\title{
OURO EM SERICITA-QUARTZO HIDROTERMALITOS CONTROLADOS POR CISALHAMENTOS CONJUGADOS BRASILIANOS, NA REGIÃO DE CAVALCANTE (GO) .BRASIL CENTRAL
}

\author{
LUIZ JOSÉ HOMEM D'EL-REY SILVA \& VIRDÁLIO SENNA FILHO
}

\begin{abstract}
GOLD IN SERICITE-QUARTZ HYDROTHERMALITES CONTROLED BY BRASILIANO CONJUGATE SHEAR ZONES IN THE CAVALCANTE (GQ) REGION, CENTRAL, BRAZIL In the Cavalcante area, north-northeastern part of the Goiás State, Central Brazil (coordinates $47^{\circ} 30-47^{\circ} 20 \mathrm{~W}$ and $13^{\circ} 40-13^{\circ} 50 \mathrm{~S}$ ), about eighty lens-shaped bodies of sericite-quartz S-C mylonites have been mapped and most of them are along dextral, ENE- and sinistrai WNW-trending, sub-vertical, ductile-brittle shear zones that intercept each other and cross-cut both the Archean-Paleoproterozoic basement and the Mesoproterozoic Araí Group. The shear zones stretch for 1-5 km whereas the Au-bearing bodies are much shorter and have an average width about 20-30 m. Seventy of these bodies have been sampled and contain gold in variable amounts, whereas the remaining ten bodies have not been prospected yet.

The Cavalcante shear zone (dextral) is one of such structures and encloses the Cavalcante Mine, which has been producing gold (and platinum) since 1750, from underground operations in a $60 \mathrm{~m}$-long segment of the Cavalcante sericite-quartz mylonite body, which is about $500 \mathrm{~m}$-long, $20-25 \mathrm{~m}$-wide and displays a sub-vertical, $\mathrm{N} 60^{\circ}-70^{\circ} \mathrm{E}$ trending $\mathrm{C}$ foliation, and a $\mathrm{N} 10^{\circ}-30^{\circ} \mathrm{E}$ trending $\mathrm{S}$ foliation dipping around $75^{\circ}-80^{\circ}$ to ESE. Abundant geology and chemistry data from drill holes and underground galleries have allowed to state that the Cavalcante orebody is an amalgamation of several lenses displaying a map-view sigmoidal geometry with 10-20 m-long axis parallel to the $\mathrm{S}$ foliation and a 3-5 m-long short axis in the perpendicular direction. They plunge about $80^{\circ}$ to ENE and are continuous at depth for several tens of meters, as demonstrated by the mining operations. Gold occurs as generally sub-mm flattened grains controlled along the foliation planes and the higher grades are associated with ore displaying a hydrothermal alteration characterized at least by pyrite, tourmaline, magnetite and carbonate minerals. Detailed structural relationships clearly demonstrate that the mineralisation is syn-tectonic and the deformation was progressive, as Au-bearing syn-tectonic siliceous veins emplaced sub-parallel to the S-C planes or along synthetic R planes (possibly R' planes as well) were also sheared and washed by Bo-bearing fluids.

An absolute age determination of the mineralisation is still missing, but the conjugate pair of shear zones fits well with a nearly E-W trending maximum horizontal stress that is entirely compatible with the Brasiliano event already documented in the surroundings of Cavalcante. The tens of Au-bearing bodies of hydrothermalite along the shear zones places the Cavalcante area in the rank of those with a very high potential for gold exploration.
\end{abstract}

INTRODUCÃ̃O A retomada das atividades de exploração mineral no Brasil, nos últimos anos, vem sendo caracterizada pelo aporte de recursos oriundos de bolsas de valores no exterior e pelo direcionamento preferencial para áreas cujas mineralizações de ouro estejam consolidadas pelo potencial metalogenético e/ou pelas condições de alcançar níveis de produção atrativos aos novos capitais.

Neste trabalho os autores discorrem sobre a geologia e os controles estruturais regionais e de detalhe da mineralização de ouro na região de Cavalcante, situada a cerca de $300 \mathrm{~km}$ a norte do Distrito Federal (Brasília), no norte-nordeste do estado de Goiás, buscando evidenciar os elementos geológicos que caracterizam a região como altamente promissora para a exploração mineral. $\mathrm{O}$ presente trabalho baseia-se em novos dados oriundos das frentes de lavra na Mina Cavalcante, bem como de observações de campo e dados da literatura pertinente.

GEOLOGIA REGIONAL E CONTROLES DA MINERALIZAÇ̃̃O DE OURO Na região que circunda Cavalcante (Fig. 1), e regiões adjacentes a oeste (Colinas) e a leste (Terezina de Goiás), todas no contexto do segmento setentrional da Faixa Brasília, afloram rochas grani-to-gnáissicas do Complexo Basal goiano, xisto grafitoso e metavulcânica da Formação Ticunzal, além de quartzito, metaconglomerado, metavulcânica (Formação Arraias) e metasiltito, filito e metasiltito carbonático (Formação Traíras), do Grupo Araí (Fuck et al. 1988). Essa porção do estado de Goiás contém evidências de policiclismo tectônico, uma vez que as rochas acima nomeadas (Marini et ali. 1984, DNPM 1987) foram afetadas por três eventos tectônicos regionais.

O ciclo Transamazônico $(2100$ - $1800 \mathrm{Ma})$ afetou rochas provavelmente Arqueanas/Paleoproterozóicas do Complexo Basal Goiano (Fuck 1994) e também xistos grafitosos e meta vulcânicas que podem ser correlacionadas às rochas da Formação Ticunzal de Marini et al. (1978). Em conjunto, esses litotipos adquiriram a direção geral N-S e estão intrudidas (na região de Terezina de Goiás) por corpos graníticos tardi-orogenéticos potencialmente mineralizados a estanho e de idades em torno de 1800-1700 Ma (Marini e Botelho 1986, Pimentel et al. 1991).

Tais granitos coincidem com o desmantelamento da cadeia Paleoproterozóica e o início do episódio de extensão crustal que é também marcado pela intrusão dos corpos máficos-ultramáficos de Niquelância, Barro alto e Canabrava situados a oeste (Nilson et al. 1994) e pelo evento de rifteamento denominado Espinhaço (Mesoproterozóico), o qual é evidenciado pelo vulcanismo e sedimentação tipo rifte continental do Grupo Araí (Schobbenhaus 1993, 1996), que por sua vez repousa discordantemente sobre o Complexo Basal Goiano, Formação Ticunzal e os granitos estaníferos. Carecendo de um melhor con- hecimento de campo, o corpo granítico mapeado a norte de Cavalcante (Fig. I; Dyer 1985) não tem idade conhecida e é aqui admitido corno Neoproterozóico, por mera hipótese.

O Ciclo Brasiliano começou com a sedimentação dos Grupos Paranoá e Bambuí (respectivamente aflorantes bem a S e W de Cavalcante e bem a E de Terezina de Goiás) e culmi- nou com a deformação entre 650-600 Ma (Fuck 1994). Este ciclo afeta todas as rochas aqui mencionadas, segundo traba-lhos de campo realizados pelo autor principal, na região de Terezina de Goiás, como parte de um grupo de professores e formandos da graduação da UnB (dados inéditos ou em parte constantes em relatórios de graduação da UnB-IG, segundo Santos e Alves 1995, Batista e Silva 1995, Souza et al. 1995, Souza e Mello 1995, Lustosa e Lima 1995, Sabatier e Melo 1995).

Tal como observável em fotografias aéreas (PROSPEC 1967) e também em campo, as rochas da região de Cavalcante estão afetadas por dobras quilométricas a métricas com trend em geral N-S (Fig. 1) e plano axial mergulhando forte a moderadamente para W. Na região de Terezina essas dobras mesoscópicas são Brasilianas e têm eixo com caimento suave, ora para SSW ora para NNE. Num raio de até $10 \mathrm{~km}$ de distância da cidade de Cavalcante (coordenadas $47^{\circ} 30^{\prime}-47^{\circ} 20^{\prime} \mathrm{W}$ e $13^{\circ} 40^{\prime}-13^{\circ} 50^{\prime} \mathrm{S}$ ) rochas e dobras estão cortadas por uma rede de lineamentos, muitos dos quais são falhas dúcteis-rúpteis que se interceptam mutuamente e que podem ser interpretados como parte de um sistema conjugado constituído por cisalhamentos ora dextrais, sub-verticais e com direção geral $N 70^{\circ}-50^{\circ} \mathrm{E}$, ora sinistrais, sub-verticais e com direção geral N60 $-40^{\circ} \mathrm{W}$. Cisalhamentos com as duas direç̃es e respectivas cinemáticas afetam ortognaisses dobrados do Complexo Basal Goiano no afloramento de dimensões decamétricas no leito do rio das Almas, sob a ponte da estrada pavimentada Cavalcante -Terezina (Fig. 1).

De um modo geral os cisalhamentos são quilométricos e controlam cerca de 80 corpos alongados e resistentes que se destacam na topografia local. Os lineamentos que contêm os corpos podem ser vistos na paisagem a partir dos pontos mais altos da região. Tais corpos, que foram cartografados pela INTEC SÁ, Grupo BP (Dyer 1985), têm comprimento máximo de até $3-4 \mathrm{~km}$, largura máxima possivelmente da ordem de 50 metros e a maioria se encaixa nas rochas do Complexo Basal Goiano e da Formação Ticunzal, em nível topográfico que marca a base do Grupo Araí (Fig. 1). Esse controle é provavelmente devido a que, nas áreas onde se deu a erosão do Grupo Araí, a exposição das rochas do Arqueano -Paleoproterozóico permitiu maior eficiência à erosão, enquanto os hidrotermalitos silicosos vêm, desde então, resistindo mais ao proceso.

Embora alguns dos cisalhamentos e cristas silicosas tenham direção bem próxima do norte, particularmente na parte SE da área consid- 


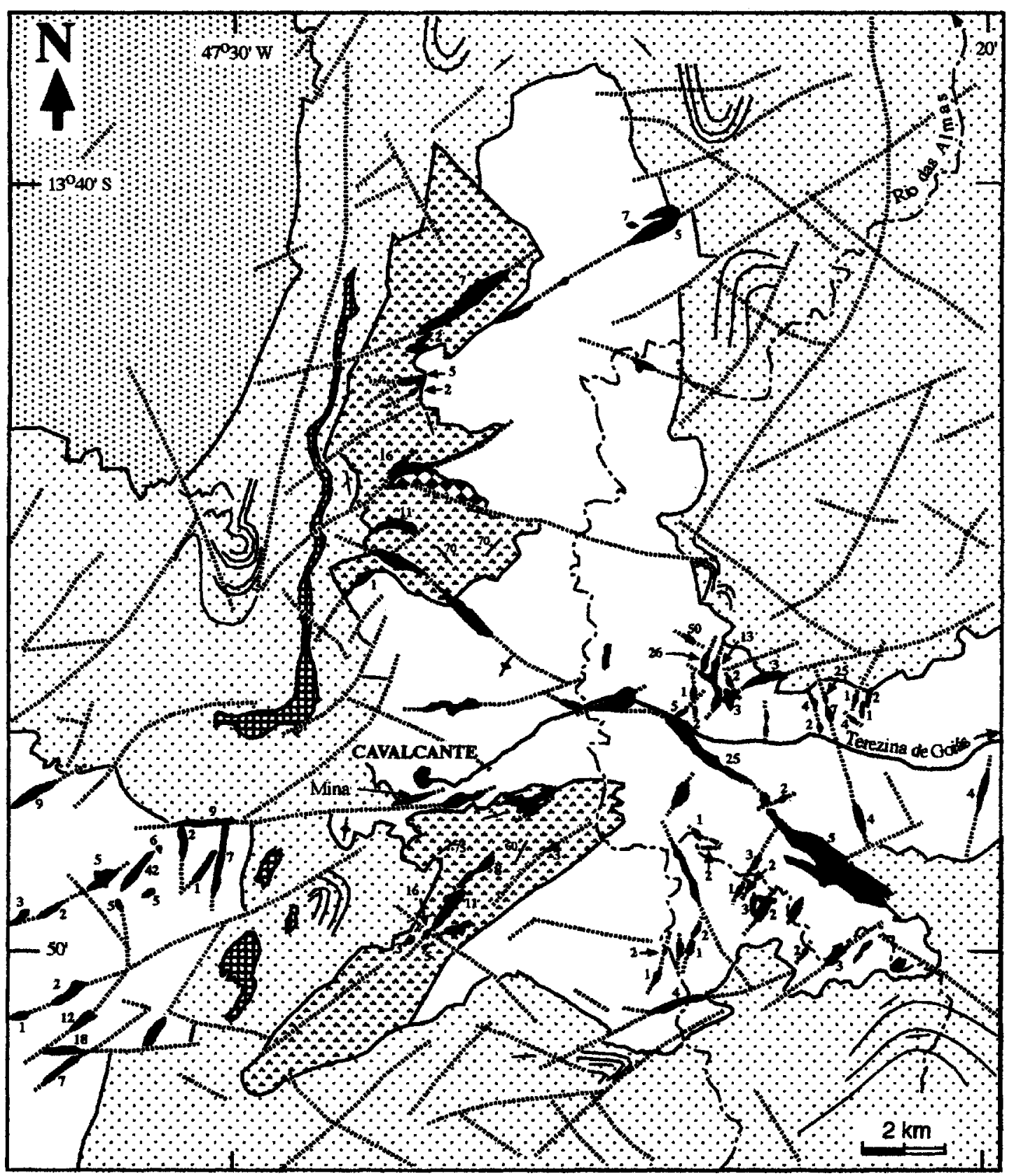

NEOPROTEROZOICO (?)

EXe Granito

MESOPROTEROZÓICO (Grupo Ará)

Formaça Trauras : metasiltito, filito,

$\because \because 1$ Formaça Arraias : quartzito, conglomerado, metavulcanica

\section{ARQUEANO - PALEOPROTEROZÓICO}

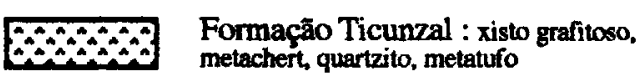

Complexo Basal Goiano : Gnaisse e ontognaisse milonfíticos munn. Lineamentos indiscriminados e zonas de cisalhamento ductil - rúptil onde ocorrem as hichotermalitos

Hidnotermalitos silicosos (milonitos $\mathrm{S}$-C) com número

maximo de pintas de ouro em solo (falta de número

5 significa valor nulo ou corpo nato amostrado)

- Acamamento observado em foto afrea ou medido em campo com valor de mergulho indicado

- Foliaçăo vertical, indiscriminada, no embasamento

A Dobras indiscriminadas observadas em foto atrea

Figura 1 - Mapa geológico simplificado da região de Cavalcante - Go, ressaltando os corpos de hidrotermalitos auriferos controlados ao longo de zonas de cisalhamento que afetam todas as rochas aflorantes (baseado no mapa 1:2.500 de Dyer 1985, simplificado e pouco modificado). A cobertura sedimentar Terciária foi omitida por simplicidade, assim como também os indicadores de cinemática das zonas de cisalhamento principais, particularmente ao longo da Mina de Cavalcante e no afloramento sob a ponte da estrada Cavalcante-Terezina, no leito do rio das Almas. 
erada, o conjunto de cisalhamentos de direções ENE e WNW é amplamente majoritário e teoricamente corresponde a um campo de tensões compressivas horizontais máximas orientadas segundo direções bem próximas de $\mathrm{E}-\mathrm{W}$, coincidindo com a orientação de $\sigma_{1}$ na região de Terezina de Goiás para o Ciclo Brasiliano (dados inéditos do autor principal ou encontrados em parte em trabalhos de Santos e Alves 1995, Batista e Silva 1995, Souza et al. 1995, Souza e Mello 1995, Lustosa e Lima 1995, Sabatier e Melo 1995).

A MINA DE CAVALCANTE Geologia básica A mina de ouro de Cavalcante é parte da zona de cisalhamento de direção ENE que tangencia o limite sul da cidade homônima e do sistema de cisalhamentos já mencionados (Fig. 1). Tais estruturas se caracterizam pela geração de rochas extremamente silicosas e micáceas finas, verdadeiros hidrotermalitos originados pela intensa percolação de fluidos que, além do ouro e da platina em si, trouxeram também o conteúdo químico de minerais como o quartzo, sericita, clorita, e dos que marcam as bordas de alteração hidrotermal, em especial prrita (arsenopirita) magnetita, turmalina e carbonates.

$\mathrm{Na}$ mina propriamente, não é possível reconhecer o protolito dos milonitos, embora exista a tentação de se considerar que sejam os quartzitos Araí, os quais afloram bem próximo em escarpas verticais. No entanto, o contato basal do Grupo Araí é sub-horizontal e situa-se topografícamente ao mesmo nível ou algo acima da crista de milonitos. De fato, segundo mapeamento geológico de detalhe (Costa \& Mozzer 1994) a encaixante dos hidrotermalitos da Mina de Cavalcante consiste de ortognaisses do Complexo Basal Goiano, enquanto que ao longo do contato entre encaixante e milonitos ocorrem tectonitos xistosos finos, os quais podem ser considerados como produtos de alteração do embasamento por efeito da deformação intensa (Massucato \& Hippertt 1996).

Pode-se então admitir que os hidrotermalitos auríferos da Mina de Cavalcante representam a faixa central longitudinal da zona de cisalhamento, justamente aquela onde se espera que tenha ocorrido a maior percolação de fluidos.

A crista de hidrotermalitos de Cavalcante apresenta, em geral muito bem desenvolvido, o típico par de foliações $\mathrm{S}-\mathrm{C}$, sendo que a foliação $\mathrm{C}$ tem direção geral equivalente à da borda do corpo $\left(\mathrm{N} 60^{\circ}-70^{\circ} \mathrm{E}, \mathrm{SV}\right)$, enquanto a foliação $\mathrm{S}$, marcada por sericita e localmente clorita, tem atitude média segundo $\mathrm{N} 10^{\circ}-20^{\circ} \mathrm{E}, 85^{\circ} \mathrm{SE}$ ou mesmo sub-vertical. Essas foliações são praticamente coincidentes em planos verticais, mas são facilmente distinguíveis em superfícies horizontais, o que sugere fortemente que as zonas de cisalhamento são de fato transcorrências. No entanto, por falta de marcadores de deformação, no interior e na crista da Mina Cavalcante, não se tem notado a lineação de estiramento sub-horizontal, embora sejam observadas raras estrias em tal posição ao longo nos planos da foliação $\mathrm{S}$ (e também C). Nesses planos as lineações mais comumente observadas são micro-crenulações downdip, em especial nas superfícies micáceas.

Entretanto, fenocristais de feldspato ocorrem elongados sub-horizontalmente no plano da foliação vertical, com sombras de pressão e pronunciado fraturamento tipo $\mathrm{T}$, inclusive com afastamento dos fragmentos no estilo pull-apart, definindo bem a lineação de estiramento de rake muito baixo em metatufos vulcânicos extremamente cisalhados da Formação Ticunzal, tanto em testemunhos de sondagem da Mina Cavalcante como em afloramentos de superfície ao longo da crista de hidrotermalitos imediatamente a leste (denominada Encantado)

Controle estrutural de detalhe Os dados de amostragem de superfície e de detalhe de lavra ao longo das galerias dos níveis 730 e 710 vêm mostrando que a mineralização ocorre em corpos sigmóides que acompanham a foliação $\mathrm{S}$, tal como já havia sido identificado por Costa \& Mozzer 1994). Entretanto, o espaçamento das lentes mineralizadas não guarda a "distância mágica" de 30 metros preconizada por aqueles autores. Os dados vêm mostrando que as lentes se dispõem lateralmente umas às outras com espaçamento horizontal da ordem de 5-8 m, e são contínuas por dezenas de metros ao longo do caimento de $75-80^{\circ}$ para ENE. Varia muito o teor de ouro nas lentes, mas há intervalos com até 100 ppm ou até mais, enquanto o teor médio dos hidrotermalitos encaixados na zona de cisalhamento, consideradas como um todo, pode ser admitido como da ordem de 4 ppm, conforme dados do segundo autor.

Nossas observações de detalhe, feitas em paredes e teto de galerias (portanto em 3 dimensões) indicam claramente que a mineralização aurífera é sintectônica e o ouro ocorre principalmente em palhetas ou grãos achatados segundo os planos da foliação, com dimensões maiores da ordem de $5 \mathrm{~mm}$. É também notado que o aporte de fluidos silicosos foi progressivo, uma vez que corpos tabulares resultaram da silica que percolou a encaixante milonítica ao longo de fraturas do tipo $\mathrm{R}$ de Riedel. Esses corpos silicosos cortam a foliação $\mathrm{S}-\mathrm{C}$ dos sericitaquartzo milonitos, são cortados por cisalhamentos discretos portadores de fluidos ricos em Boro, e foram brechados hidraulicamente por fluidos provavelmente ricos em ferro. Há, igualmente, evidências de que tais fluidos percolaram ao longo de planos de foliação $\mathrm{C}^{\prime}$ (Lister \& Snoke 1984) que se desenvolveram com a progressão da deformação.

Embora sejam requeridos trabalhos de detalhe para esclarecer se há um controle microscópico preferencial do ouro em uma das foliações (C, S, ou $\mathrm{C}^{\prime}$ ), ou se ao longo de intersecções destas, para efeito de planejamento da lavra o entendimento atual do controle espacial é suficiente, dada a escala da operação de mineração. Em adição, o entendimento do controle mesoscópico tende a ser maximizado com trabalhos de análise estrutural que serão efetuados com o avanço de lavra.

DISCUSSÃO E CONCLUSÕES O fato de que as zonas de cisalhamento cortam as dobras regionais e locais, sendo portanto feições estruturalmente tardias, e de que correspondem a um campo de tensões compatível com o do Ciclo Brasiliano na região, indicam que muito provavelmente a mineralização é de idade compatível com a do fim deste ciclo (aproximadamente $600 \mathrm{Ma}$, segundo Viana et al. 1995, Pimentel et al. 1997).

É bem possível que o ouro tenha sido lixiviado das rochas granitognássicas do embasamento (como apontam Massucato \& Hippertt 1996,1997) ou que possa também ser originado de granitos Meso e/ou Neoproterozóicos aflorantes e não aflorantes na região. De qualquer forma, em futuro próximo serão realizados estudos de detalhe utilizando inclusões fluidas em amostras de rochas coletadas com rígido controle estrutural, em galeria da Mina Cavalcante, visando ao melhor entendimento da origem das soluções mineralizantes.

A ocorrência dos corpos Au-silicosos como lentes isoladas ao longo das zonas de cisalhamento ainda não está bem entendida. Esse controle pode significar que os hidrotermalitos resultam do acúmulo de fluidos em zonas de baixa pressão nos cisalhamentos regionais, isto é, em prováveis sinuosidades, ainda que sutis, ao longo do traço da falha, levando à formação de releasing bends (Christie-Blick \& Biddle 1985). Em tais sinuosidades a deformação cisalhante pode ser do tipo oblíqua - divergente (Sanderson \& Marchini 1984, Krantz 1995) o que favorece a criação de vazios simultâneos com a deformação. Esta possibilidade teórica ainda requer demonstração, porém é mais atraente que as demais alternativas de que os corpos coincidiriam com intrusões graníticas ao longo das zonas de cisalhamento em profundidade ou, então, a localização dos corpos mineralisados deve ser encarada como aleatória ao longo das falhas.

Os dados regionais de controle das cristas de hidrotermalitos e os dados de detalhe no interior da Mina de Cavalcante são inteiramente contrários à interpretação de que a mineralização aurífera da região foi controlada por fraturas extensionais (gashes) de escala quilométrica e integrantes de um cisalhamento NE-SW de escala crustal, como preconizado por Massucatto \& Hippertt (1996,1997), Hippert \& Massucatto (1998). As cristas auríferas de Cavalcante são em si mesmas típicas zonas de cisalhamento ao longo das quais se desenvolveram milonitos S-C e como tal devem ser descritas, não podendo ser interpretadas como tension gashes por razões conceituais básicas.

Unicamente a bem do conhecimento da geologia da região, outros aspectos desse trabalho devem ser debatidos: 1 - Não existem evidências de campo para a suposta zona de cisalhamento dúctil de escala crustal, na região de Cavalcante e Terezina de Goiás; 2 - As cristas mineralizadas a ouro não são transversalmente cortadas por zonas de cisalhamento, como aponta a figura 7 de Hippert \& Massucatto (1998); 3 - Ainda que fosse o caso, estruturas tipo tension gashes geradas em uma zona de cisalhamento não seriam transformadas em zonas de cisalhamento S-C paralelas ao maior eixo das próprias gashes como pretendem Massucatto \& Hippertt $(1996,1997)$. Elas poderiam ser rotacionadas e dobradas em estágios seguintes da deformação progressiva, e até ser truncadas por cisalhamentos desenvolvidos ao longo dos planos R, R', P e X da zona de cisalhamento. Mesmo assim, tudo isto implicaria em bulk strain acentuado, incompatível com a posição real das cristas mineraliza-das dentro da suposta zona de cisalhamento de escala crustal.

Mesmo em menor escala não se notam fraturas extensionais do tipo $\mathrm{T}$ nas frentes de galeria nem nos afloramentos de superfície, na crista de Cavalcante. Em superfície são observadas algumas poucas fraturas extensionais de baixo ângulo de mergulho, preenchidas por veios de comprimento métrico e espessura decimétrica, de quartzo leitoso. Tais 
feições são bastante diferentes dos supostos gashes tanto em escala como em função do sistema cisalhante, e não se tem notícia de que sejam portadoras preferenciais de ouro.

Os controles estruturais da mineralização em Cavalcante, tais como descritos neste breve artigo, implicam em não haver regiões mais ou menos favorecidas para o acúmulo de ouro em cada corpo de hidrotermalito, visto que as foliações ocupam todo o corpo de rocha silicosa (informações abundantes obtidas nas galerias, no campo, e também no mapa de Costa \& Mozzer 1994). Isso implica que o corpo silicoso da Mina pode conter lentes mineralizadas ao longo de todo o seu comprimento (quase $\mathrm{E}-\mathrm{W}$ ), o que, sem duvida, é fator determinante do potencial de reservas de ouro no local e também regionalmente. Sondagens rotativas a diamante atualmente em execução na Mina Cavalcante vêm demonstrando a veracidade dessa premissa geológica, implicando em consi-derável aumento das reservas conhecidas até seis meses atrás. A economicidade do depósito mineral pode vir a ser reforçada mais ainda pela ocorrência subordinada de platina que, embora já tenha sido detectada por Marchetto et al. (1993), não constituiu objeto de análise no presente estudo.

$\mathrm{O}$ fato de que as cristas de hidrotermalitos auríferos só afloram entre rochas do embasamento, estando quase sempre no nível topográfico da base do Grupo Araí, implica que os lineamentos que atravessam a Formação Arraias (Fig. 1) podem igualmente encaixar corpos de hidrotermalitos, em profundidade compatível com aquele nível, ou até mesmo mais próximos da superfície.

Agradecimentos - À Mineração Cavalcante pelo apoio na realização dos trabalhos de campo. L.J.H. D'el-Rey Silva agradece aos engenheiros Annibal Crosara e Orlando Generoso (Penery Mineração, Grupo EMSA, Goiânia), ao Dr. Eduardo Passos (Mineração Cavalcante) e ao CNPq (Processo 520.241/95-9).

\section{Referências}

Batista, LM. e Silva, C.C.M. da 1995. Projeto Cavalcante, área II. Relatório de Graduação em Geologia, UnB-IG, 85 p., inédito.

Costa, J.L.G. e Mozzer, F. 1994. As mineralizações de ouro e platina da Mina Buraco do Ouro, Cavalcante, Goiás, aspectos geológicos e estruturais. Relatório interno da Mineração Cavalcante, 4 páginas e 4 figuras.

Christie-Blick, N. and Biddle, K.T. 1985. Deformation and basin formation along strike-slip faults. Society of Economic Paleontologists and Mineralogists (SEPM) Bulletin 1, $1-33$

DNPM 1987. Mapa geológico do estado de Goiás, escala 1:1.000.000.

Dyer, R.C. 1985. Projeto Cavalcante. Relatório Preliminar de Pesquisa realizada pela INTEC SÁ para o DNPM 860.523/81, contendo 19 páginas e o mapa geológico das malhas $1^{\mathrm{A}}, 1 \mathrm{e} 2$, escala 1:2.500. Documento interno da Mineração Cavalcante

Fuck, R. A. 1994. A Faixa Brasília e a compartimentação tectônica da Província Tocantins. In IV Simpósio Geologia do Centro Oeste, Anais SBG Centro Oeste, 184-187.

Fuck, R.A, Marini, O.J., Dardenne, M.A. e Figueiredo, NA.F. 1988. Coberturas metassedimentares do Proterozóico Médio: Os Grupos Araí e Paranoá na região de Niquelândia-Colinas, Goiás. Revista Brasileira de Geociências 18(1), 54-62.

Hippertt, J.F. and Massucatto, J. 1998. Phyllonitization and development of kilometer-scale extension gashes in a continental-scale strike-slip shear zone, north Goiás, central Brazil. Journal of Structural Geology 20(4), 433-445.

Krantz, R.W. 1995. The transpressional strain model applied to strike-slip, oblique-convergent and oblique-divergent deformation. Journal of Structural Geology 17 (8), 1125-1138.

Lister and Snoke 1984. S-C mylonites. Journal of Structural Geology 6,617-638.

Lustosa, G.P. e Lima, A.B. de 1995. Projeto Cavalcante, área IV. Relatório de Graduação em Geologia, UnB-IG, 92 p., inédito.

Marchetto, C.M.L., Larson, L.T. e Figueiredo, A.M. de 1993. Minerais do Grupo da Platina e Ouro em rochas ácidas, Cavalcante, Brasil. I Encontro Brasileiro de Elementos do Grupo da Platina, Anais, 47-52.

Marini, O.J. e Botelho, N.F. 1986. A província de granitos estaníferos de Goiás. Revista Brasileira de Geociências 16(1), 119-131

Marini, O.J., Fuck, R.A., Dardenne, M.A, Danni, J.C.M. 1984. Província Tocantins, Setores Central e Sudeste. In F.F.M. de Almeida e Y. Hasui (Coordenadores) O Pré-Cambriano do Brasil, Edgard Blücher editora, 205-264.

Marini, O.J., Liberal, C.S., Reis, L.T., Trindade, C. Al.H. e Souza, C.L. 1978. Nova Unidade Litoestratigráfica do Precambriano do Estado de Goiás. In XXX Congresso Brasileiro de Geologia, Recife, Boletim Especial SBG 1, 126-127.

Massucato, A.J. e Hippert, J.F.M. 1996. Mineralização aurífera associada a estruturas S-C em "gashes" extensionais de escala quilometrica - o exemplo de Cavalcante - Go. In XXXIX Congresso Brasileiro de Geologia, Salvador, Anais SBG 1, 343-346.
Massucato, A.J. e Hippert, J.F.M. 1997. Geração e obliteração de microestruturas de "crack sealing" em veios de quartzo de dimensões quilométricas (Cavalcante, GO). In VI SNET - Simpósio Nacional de Estudos Tectônicos, Pirinópolis-Go, Anais SBG, 193-195.

Nilson, A.A., Botelho, N.F. e Ferreira Filho, C.F. 1994. Rifteamento crustal Meso-Proterozóico no centro-norte de Goiás. In XXXVIII Congresso Brasileiro de Geologia, Camboriú, Anais SBG, 1, Simpósios, 258-259.

Pimentel, M.M., Heaman, L., Fuck, R.A. and Marini, O.J. 1991. U-Pb zircon central Brazil. Precambrian Research 52,321-335.

Pimentel, M.M., Whitehouse, M.J., Viana, M. das G., Fuck, R.A. and Machado, N. 1997. The Mara Rosa Arc in the Tocantins Province: further evidence for crustal accretion in Central Brazil. Precambrian Research 81, 299-310.

PROSPEC 1967 - Fotos aéreas na escala 1:45.000, (226-1309A) números 4953 a 4956 (226-1310A) números 3579 a 3582; e (226-1308A) números 5460 a 5463.

Sabatier, S.F. e Melo, M.V.L.C. de 1995. Projeto Cavalcante, área V. Relatório de Graduação em Geologia, UnB-IG, 90 p., inédito.

Sanderson, D.J. and Marchini, W.R.D. 1984. Transpression. Journal of Structural Geology $6,449-458$.

Santos, P.M.C. dos e Alves, R, de L. 1995. Projeto Cavalcante, área I. Relatório de Graduação em Geologia, UnB-IG, 90 p., inédito.

Schobbenhaus, C. 1993. O Proterozóico Médio no Brasil com ênfase à Região Centro-Oeste. Tese de Doutorado, Freiburg in Breisgau - Alemanha, 166p.

Schobbenhaus, C. 1996. As tafrogêneses superpostas espinhaço e Santo Onofre, estado da Bahia: revisão e novas propostas. Revista Brasileira de Geociências 26(4), 265-276.

Souza, C. S. de, Matos, J.M. da S. e Kitajima, L.F.W. 1995. Projeto Cavalcante, área III Relatório de Graduação em Geologia, UnB-IG, 85p., inédito.

Souza, M.T. e Mello, G. de A. 1995. Projeto Cavalcante, área VI. Relatório de Graduação em Geologia, UnB-IG, 83 p., inédito.

Viana, M. das G., Pimentel, M.M., Whitehouse, M.J., Fuck, R.A. and Machado, N. 1995. The Mara Rosa magmatic are, Goiás: geochemical and geochronological data and their regional significance. Revista Brasileira de Geociências 25 (2), 111-123.

Manuscrito NB-16 Recebido em 29 de abril de 1998 Revisão dos autores em 10 de maio de 1998 Revisão aceita em 12 de maio de 1998 geochronology of Precambrian tin-bearing continental-type acid magmatism in 